\title{
REORGANIZAÇÃO DAS ATIVIDADES EXTENSIONISTAS EM SAÚDE BUCAL PARA PRÉ-ESCOLARES NO CONTEXTO DA COVID-19
}

\section{THE REORGANIZATION OF THE OUTREACH ACTIVITIES IN ORAL HEALTH FOR PRESCHOOLS CHILDREN IN THE CONTEXT OF COVID-19}

\author{
Cibele Silva Barbosa* \\ ORCID: https://orcid.org/0000-0002-3352-7058 \\ Gabriela Fleury Seixas** \\ ORCID: https://orcid.org/0000-0002-2739-0307 \\ Camilla Helena Volpato*** \\ ORCID: https://orcid.org/0000-0001-8501-5540 \\ Jessica Angie Sovinski**** \\ ORCID: https://orcid.org/0000-0002-3383-6897 \\ Caio Rafael Schavarski***** \\ ORCID: https://orcid.org/0000-0002-9368-3175 \\ Cássia Cilene Dezan Garbellini****** \\ ORCID: https://orcid.org/0000-0002-8660-0677
}

\section{Resumo}

O projeto "Ações odontológicas educativas, preventivas e curativas em crianças de 0 a 6 anos atendidas no Centro Municipal de Educação Infantil (CMEI) Valéria Veronesi, Londrina” da Universidade Estadual de Londrina, tem como meta promover saúde bucal para crianças atendidas no CMEI e realizar o tratamento das necessidades odontológicas acumuladas nessa população. Diante do contexto de COVID-19, as atividades presenciais sofreram modificações para assegurar atendimento à comunidade. Logo, o objetivo deste trabalho é relatar as atividades promovidas pelo projeto antes e durante a pandemia, e apresentar as propostas desenvolvidas pelos graduandos em Odontologia, residentes e professores de odontopediatria. Mesmo neste período de incertezas, o projeto contribuiu para disseminar conhecimentos, gerando melhor qualidade de vida às crianças e seus familiares, além de possibilitar que os acadêmicos desenvolvessem habilidades para atuar frente à realidade da sociedade.

Palavras-chave: Promoção da Saúde; Educação em Saúde; Pré-Escolar; COVID-19.

\begin{abstract}
The project "Educational, preventive and curative dental actions for children aged 0 to 6 years old that attend at the Municipal Center for Child Education Valéria Veronesi (CMEI, Brazilian abbreviation), Londrina", carried out by the State University of Londrina, aims to promote oral health for children assisted by CMEI and provide treatment for their dental needs. In the context of COVID-19, face-to-face activities had to undergo modifications to ensure community service. Therefore, the objective of this study is to report the activities promoted by the project before and during the pandemic period and to present the proposals outlined by undergraduate dentistry students, residents, and pediatric dentistry professors. Even in this period of uncertainty, the project contributed to disseminate knowledge, and provide a better quality of life for children and their families, in addition to enable students to develop skills to deal with social reality.
\end{abstract}

Keywords: Health Promotion; Health Education; Child, Preschool; COVID-19.

Data recebimento: $30 / 08 / 2021$

Data de aceite: $03 / 11 / / 2021$
* Aluna de Graduação da Universidade Estadual de Londrina (UEL), Londrina - PR, Brasil. E-mail: cibele.barbosa@uel.br ** Professora da Universidade Estadual de Londrina (UEL), Londrina - PR, Brasil. E-mail: gabriela.fleury@uel.br *** Aluna de Graduação da Universidade Estadual de Londrina (UEL), Londrina - PR, Brasil. E-mail: camilla_volpato@hotmail.com **** Graduação em Odontologia. Universidade Estadual de Londrina (UEL), Londrina - PR, Brasil. E-mail: jessica.angie.sovinski@uel.br ***** Aluno de Mestrado da Universidade Federal de Minas Gerais (UFMG), Belo Horizonte - MG, Brasil. E-mail: caioschavarski@gmail.com ****** Professora da Universidade Estadual de Londrina (UEL), Londrina - PR, Brasil. E-mail: cassiadg@uel.br 


\section{Introdução}

O Projeto de extensão "Ações odontológicas educativas, preventivas e curativas em crianças de 0 a 6 anos atendidas no Centro Municipal de Educação Infantil Valéria Veronesi, Londrina" foi desenvolvido em 2018, e atualmente envolve os departamentos de Odontologia Restauradora (ODO) e Medicina Oral e Odontologia Infantil (MOOI) da Universidade Estadual de Londrina (UEL). A concepção desse projeto surgiu a partir de uma estimativa rápida da condição de saúde bucal das crianças atendidas pelo Centro Municipal de Educação Infantil (CMEI) Valéria Veronesi, em Londrina-PR, na qual foi verificada a necessidade de tratamento odontológico curativo em várias crianças, principalmente devido à cárie dentária. Desde então, as atividades realizadas pelo projeto de extensão buscam promover saúde bucal com ações educativas e preventivas, e tratar as necessidades odontológicas acumuladas nas crianças atendidas pelo CMEI.

As escolas de educação infantil são consideradas espaços ideais para o estabelecimento de ações coletivas ao envolver não somente crianças, mas também pais/responsáveis e professores (SIGAUD et al., 2017). Em vista disso, de modo a promover comportamentos saudáveis na prevenção de doenças e desordens bucais, dentre as ações executadas pelo projeto estão: entrevistas com os pais e oficinas com os professores, desenvolvimento de atividades lúdicas com as crianças com intuito de ampliar o conhecimento sobre o desenvolvimento das principais patologias bucais e incentivar o autocuidado, instrução de higiene bucal, entrega de kits de higiene bucal, escovação supervisionada, exame físico e atendimento odontológico quando identificada a necessidade.

Em dezembro de 2019, porém, uma nova doença foi identificada (COVID-19), com grande potencial de transmissibilidade e mortalidade, o que modificou sobremaneira as atividades escolares e de atendimento odontológico na esfera pública. Declarada em 11 de março de 2020 como pandemia, pela Organização Mundial da Saúde (OMS), a infecção com transmissão respiratória pelo novo coronavírus (SARS-CoV-2) leva a um quadro semelhante ao da gripe e, em alguns casos, à síndrome respiratória aguda grave (WIERSINGA et al., 2020).

Por conseguinte, muitos países buscaram estratégias visando reduzir a transmissão do vírus e frear a rápida evolução da doença, que ainda não apresenta tratamento eficaz (KUPFERSCHMIDT; COHEN, 2020). Em vista disso, o Ministério da Educação (MEC) publicou, em 17 de março de 2020, a Portaria $\mathrm{n}^{\mathrm{o}} 343$, substituindo, em caráter emergencial, as aulas presenciais pelo ensino a distância $(\mathrm{EaD})$, atendendo à solicitação feita pela Associação Brasileira de Mantenedoras de Ensino Superior (ABMES) e pelo Conselho Nacional de Educação (CNE), visando evitar o descontrole da pandemia e transmissão local (BRASIL, 2020; MINISTÉRIO DA SAÚDE, 2020). 
Com o isolamento físico imposto, observou-se um grande comprometimento das ações de extensão (OLIVEIRA; DEGGAU, 2021), que é um dos pilares da educação universitária e objetiva também o desenvolvimento social dos futuros profissionais e da comunidade em que se encontra. Diante dos novos desafios que se estruturaram, foram exigidas mudanças organizacionais e tornou-se necessária a revisão das práticas coletivas em saúde bucal até então executadas, com o objetivo de desenvolver estratégias que garantissem a continuidade e manutenção do cuidado em saúde das crianças assistidas pela CMEI (DE MELO et al., 2020; MERCURI et al., 2021; CAMPOS et al., 2020). Frente à realidade da pandemia, as ações de promoção de saúde bucal do projeto de extensão foram reestruturadas, a fim de dar continuidade às atividades e assistir à população.

O objetivo do presente trabalho é relatar ações promovidas pelo projeto de extensão no CMEI Valéria Veronesi de Londrina-PR, executadas antes e durante a pandemia da COVID-19.

\section{Metodologia}

Trata-se de estudo um descritivo tipo relato de experiência das ações promovidas pelo projeto "Ações odontológicas educativas, preventivas e curativas em crianças de 0 a 6 anos atendidas no Centro Municipal de Educação Infantil Valéria Veronesi, Londrina".

As atividades foram desenvolvidas na própria CMEI e na Clínica de Especialidades Infantis/Bebê-Clínica (UEL), nos anos de 2018 a 2021. Para a condução das ações durante esse período, participaram 13 graduandos em Odontologia, do primeiro ao quinto ano do Curso de Odontologia - UEL, 9 residentes em Odontopediatria, 6 docentes de Odontopediatria e 1 docente de Odontologia restauradora. A população alvo do projeto é composta por três grupos: crianças matriculadas na CMEI, pais/responsáveis pelas crianças e professores.

\section{Relato de experiência}

\section{Ações Promovidas nos anos de 2018-2020, previamente à pandemia de COVID-19}

No início dos anos letivos de 2018 e 2019, os acadêmicos participantes do projeto receberam capacitação para execução das atividades propostas, por meio de encontros e rodas de conversa promovidas pelos docentes. Os pais ou responsáveis das crianças matriculadas na referida CMEI foram informados sobre os objetivos e a metodologia do projeto, e assinaram um termo de consentimento autorizando a participação da criança, a cada ano. O cronograma com todas as datas das visitas mensais foi enviado à direção da CMEI. 
A abordagem aos pré-escolares foi organizada de acordo com a faixa etária das turmas do CMEI, que no início de 2019 atendia 420 crianças. Apesar do período pandêmico, os pais mantiveram as crianças matriculadas, para garantir a vaga no retorno das atividades presenciais, e por isso não houve redução no número de crianças atendidas.

No berçário, as ações ocorreram da seguinte maneira: primeiramente, as crianças foram colocadas na macri (maca odontológica para bebês e crianças), destinada ao atendimento de crianças até 2 anos, onde realizou-se a limpeza da cavidade bucal, com gaze umidificada em solução de digluconato de clorexidina $0,12 \%$ nos bebês que não apresentavam dentes, ou com escova com cerdas macias e creme dental fluoretado para os dentados. Em seguida, foi realizado exame bucal e observação da necessidade de aprimoramento da higienização pelos pais, e se havia a precisão de intervenção odontológica por presença de patologia bucal. Caso houvesse necessidade de comunicação com os pais, os acadêmicos deixavam registrado na agenda escolar a orientação dos procedimentos a serem realizados em casa ou a necessidade do atendimento odontopediátrico, com encaminhamento sugerido para a Clínica de Especialidades Infantis/Bebê-Clínica UEL para o atendimento especializado. Para que o tratamento curativo fosse realizado, os pais ou responsáveis deveriam comparecer ao primeiro atendimento agendado, para obtenção da autorização do tratamento e esclarecimentos sobre os procedimentos a serem realizados. $\mathrm{O}$ agendamento dos procedimentos odontológicos não necessariamente ocorreu no período escolar, para não prejudicar o bom andamento do ensino.

Para as turmas com a faixa etária maior ( 2 a 5 anos), foi realizada a orientação de higiene bucal, utilizando-se demonstração com macromodelos. Após a instrução, cada criança recebeu um kit de higiene bucal (escova dental e dentifrício fluoretado), e realizou-se a escovação supervisionada individual e coletiva (Figura 1A), nos banheiros adaptados para crianças, que são dispostos a cada duas salas, no prédio da CMEI. Em seguida, em grupos pequenos, as crianças foram direcionadas ao consultório odontológico da Bebê Clínica, onde realizou-se o exame bucal na cadeira odontológica (Figura 1B). A Bebê Clínica e a CMEI ocupam um prédio denominado "Cidade da Criança", o qual está localizado no centro histórico da cidade, a 150 metros de distância do terminal de ônibus urbano. As unidades, embora autônomas, apresentam possibilidade de acesso às instalações de forma interna, sem que haja a necessidade de saída das crianças do complexo. Para os procedimentos de avaliação e prevenção, as crianças eram levadas à Bebê Clínica pelos alunos participantes do projeto. Da mesma forma que com a turma do berçário, a comunicação com os pais ou responsáveis para a resolução de um possível problema bucal ocorreu através de bilhetes enviados pela agenda escolar.

$\mathrm{O}$ atendimento na Bebê Clínica (Figura $1 \mathrm{C}$ ) ocorreu concomitante às outras atividades realizadas no local. Os responsáveis levavam as crianças previamente triadas para o atendimento odontológico, a ser realizado pelos residentes de Odontopediatria, os quais eram auxiliados por graduandos em Odontologia, sob supervisão dos docentes. Durante os atendi- 
mentos odontológicos, foram realizados os procedimentos necessários para cada criança, como radiografias, aplicação tópica de flúor (ATF), restauração atraumática (ART), restaurações dentárias, tratamento endodôntico, entre outros. No dia do atendimento, os pais receberam orientações sobre a condição bucal de seus filhos, métodos adequados de higiene bucal, orientação de dieta e consequências da manutenção de hábitos bucais deletérios.

As atividades lúdico-recreativas foram realizadas com todas as crianças, por meio de dinâmicas com desenhos, macromodelos, teatro com personagens e músicas com os seguintes temas: alimentação saudável, técnicas de higiene bucal, uso do fio dental e cárie dentária. Em um dos encontros, foi apresentado o Teatro "A fada do Dente e o Dentão" (Figura 1D), que envolveu também outro projeto de extensão "Projeto Saúde Bucal em Escolares e a Comunidade".

Figura 1 - Ações desenvolvidas pelo projeto de extensão. A) Escovação supervisionada. B) Exame bucal. C) Atendimento Odontológico na Bebê/Clínica. D) Teatro “A Fada do Dente e o Dentão".

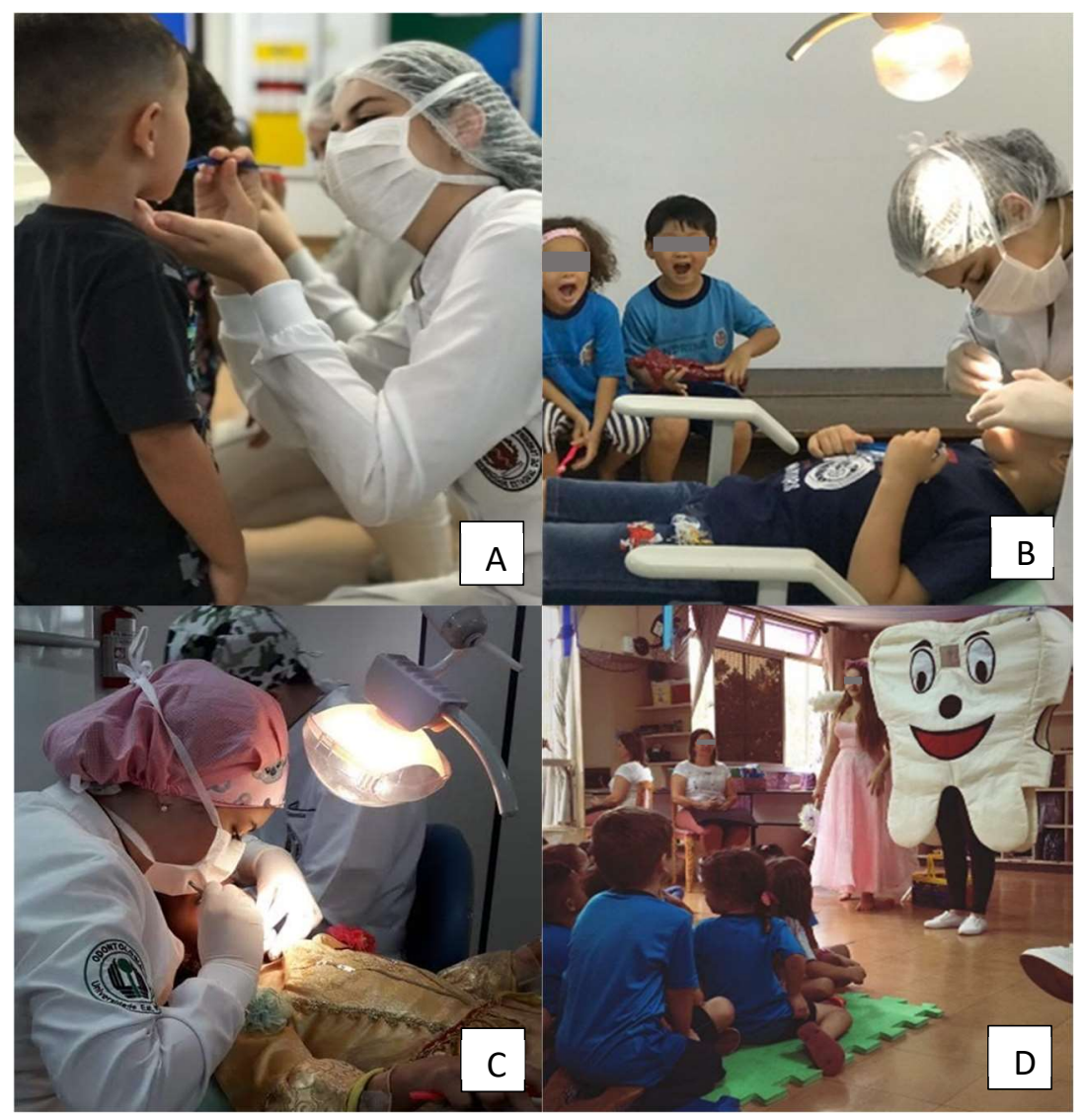

Fonte: Do autor, 2019. 
As ações com os professores contaram com oficinas e rodas de conversa sobre a importância da saúde bucal no desenvolvimento da criança, e o papel dos professores e da escola nesse contexto. A discussão com os professores envolveu orientações sobre traumatismo dentário e como proceder em caso de dor dentária relatada pelas crianças durante as atividades escolares. A escola participa do desenvolvimento da saúde da criança incentivando hábitos de dieta e higiene, e os professores da CMEI participante do projeto continuamente trabalham o tema da saúde bucal dentro dos cuidados e desenvolvimento da criança, de acordo com a metodologia empregada em sala de aula e faixa etária das crianças envolvidas.

O projeto também procurou envolver os pais e/ou responsáveis, através dos comunicados via agenda escolar, realizando orientações quanto à saúde bucal das crianças na recepção da CMEI, em horário de entrada e saída das crianças.

\section{Ações promovidas nos anos de 2020-2021, durante a pandemia de COVID-19}

Com a declaração de pandemia pela Organização Mundial de Saúde em março de 2020, as atividades odontológicas eletivas foram suspensas nos estabelecimentos públicos de saúde. Os centros de educação públicos também tiveram suas atividades presenciais paralisadas, impedindo, dessa maneira, a continuidade do projeto de extensão dentro da CMEI nos moldes em que ocorria.

Para amparar as crianças que porventura necessitassem de atendimento odontológico durante esse período, o projeto de extensão divulgou, via redes sociais, a possibilidade de atendimento de urgência e emergência odontológica realizado pela Bebê Clínica da UEL.

Percebendo-se a necessidade de criar alguma atividade que se ajustasse à nova rotina imposta pelo isolamento social, as ações educativas e preventivas ocorreram por meio da criação de vídeos e cartilhas, pelos alunos participantes. Esses materiais foram direcionados aos pais e às crianças, através da coordenação da CMEI, e encaminhados via WhatsApp aos responsáveis. Alguns materiais produzidos pelos alunos participantes podem ser visualizados acessando o QR Code disponível a seguir (Figuras 2 e 3). De acordo com Melo, Fonseca e Vasconcellos-Silva (2017), as ferramentas tecnológicas conseguem atingir diferentes grupos sociais, permitindo o acesso e compartilhamento rápido e eficiente das informações. 
Figura 2 - $Q R$ Code de um dos vídeos desenvolvidos pelo projeto.

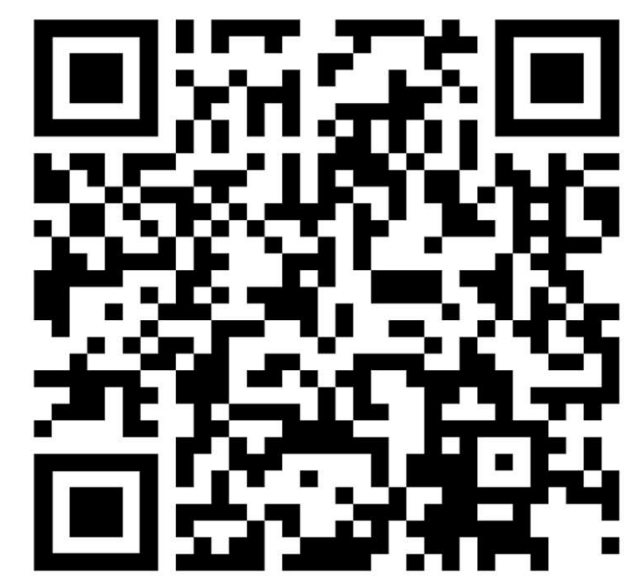

Fonte: Do autor, 2021

Figura 3 - QR Code para acesso à cartilha "Promoção de saúde bucal na infância".

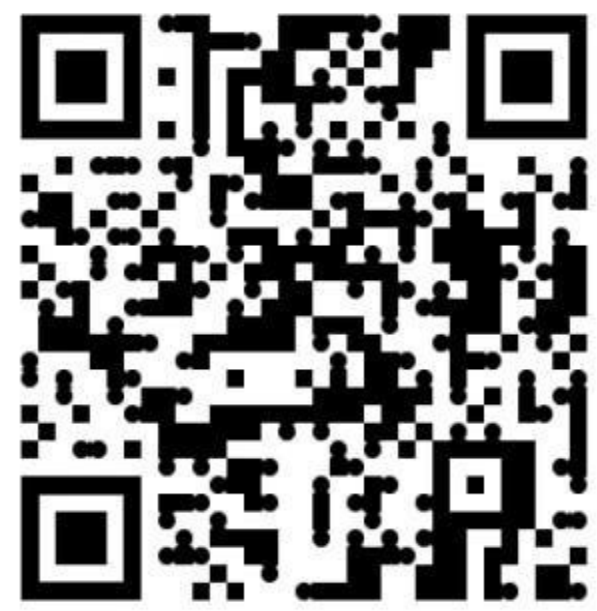

Fonte: Do autor, 2021 
As produções audiovisuais (vídeos) trouxeram informações breves, de no máximo 4 minutos, com o objetivo de transmitir orientações e permitir a compreensão rápida do comteúdo, com o uso de linguagem de fácil entendimento. $\mathrm{O}$ material didático direcionado aos pais apresentou os temas: dieta saudável e consumo controlado de doces, higiene bucal, traumatismo dentário e hábitos bucais deletérios. Para as crianças, foi divulgado um vídeo em que super-heróis realizam a escovação dentária com uma música de fundo, de modo a ser utilizado como motivador durante a higiene bucal do pré-escolar.

Foi compartilhada com os pais, também, uma cartilha com as informações detalhadas sobre os temas abordados nos vídeos, de maneira ilustrada e simplificada. Ao final de cada material, foi disponibilizada uma mensagem incentivando o público a mandar possíveis dúvidas ou sugestões para o e-mail do projeto.

\section{Resultados}

Durante os anos de 2018 a 2020, foram atendidas anualmente 1.226 pessoas, sendo 420 crianças de 0 a 5 anos, o que corresponde ao número total de vagas ofertadas anualmente, 716 pais/responsáveis (mínimo estimado) e 104 professores do CMEI. No período pré-pandêmico, mediante as ações realizadas presencialmente, foi possível observar que as crianças demonstraram grande interesse com as atividades desenvolvidas, impactando positivamente na melhora e manutenção da saúde bucal. Os professores foram capacitados para atuar diante situações de urgência odontológica na escola, e os pais mostraram ter maior interesse e conhecimento sobre a condição de saúde bucal de seus filhos. A instrução de higiene bucal foi realizada mensalmente para todas as crianças, perfazendo um total de cerca de 4.000 orientações ao ano. Aproximadamente 150 crianças apresentavam experiência de cárie dentária em graus variados, a maioria passível de controle por técnicas minimamente invasivas (diaminofluoreto de prata, tratamento restaurador atraumático, modificação de fatores predisponentes). Em um grupo reduzido, foi identificada a necessidade de tratamento restaurador convencional, endodontias, exodontias e mantenedores de espaço.

O fato de ambas as unidades compartilharem o mesmo prédio facilitou sobremaneira o atendimento de urgências durante o período pré-pandemia; os professores levavam as crianças que se queixassem de dor nos dentes/boca ou aquelas que sofriam traumatismo dentário durante o período escolar para a Bebê Clínica, onde eram prontamente atendidas. Durante a pandemia, os pais foram orientados a buscarem o atendimento de urgência, caso necessário, em Unidades Básicas de Saúde ou no Pronto Atendimento da Bebê Clínica. 
Notou-se a importância das orientações e práticas relacionadas à educação em saúde no CMEI. Para uma grande parcela das crianças, o projeto foi o primeiro contato com cirurgiões-dentistas, verificando-se posteriormente, no atendimento odontológico, um comportamento mais positivo diante dos procedimentos em cadeira odontológica, refletindo menor ansiedade.

No contexto imposto de isolamento social, os materiais desenvolvidos na modalidade virtual colaboraram com a continuidade da educação interprofissional e contribuíram com o fortalecimento das estratégias de prevenção, além da promoção de saúde das crianças e famílias durante a pandemia. O uso das tecnologias na comunicação com a família tornou-se um importante aliado para a disseminação de boas práticas em saúde e deverá permanecer como estratégia de incentivo à manutenção da qualidade de vida, mesmo após o retorno às atividades presenciais das escolas de educação infantil.

\section{Discussão}

A desinformação de pais e crianças sobre os fatores causais das doenças bucais, assim como da importância da correta higiene bucal, adoção de dieta saudável e o papel dos dentes decíduos no desenvolvimento normal da arcada contribuem para a instalação de problemas bucais (LAI et al., 2017; MONTES, 2019). Um baixo grau de "alfabetização em saúde bucal" se mostrou um fator de risco para o desenvolvimento de cárie nos dentes decíduos, quando o responsável não se percebe como determinante do processo saúde/doença de seu filho, atribuindo a outros a responsabilidade desses cuidados (NUNES; PEROSA, 2017; FIRMINO et al., 2018; MONTES et al., 2019).

As instituições de educação infantil são espaços privilegiados para o desenvolvimento de ações extensionistas, pois é na infância que a criança aprende e incorpora hábitos de cuidado quando motivadas, tornando esse comportamento resistente a mudanças, além de permitir que as atividades se estendam aos pais e professores (SIGAUD et al., 2017; MONTEIRO; CASTRO, 2021). Nunes et al. (2019) descreveram que a introdução de temas relacionados à saúde bucal para crianças, pais/responsáveis e educadores resulta na prevenção de doenças bucais, especificamente quando as atividades são trabalhadas com crianças na faixa etária até os 6 anos de idade.

Quanto às ações voltadas aos profissionais da educação, observa-se uma grande importância na realização de capacitação e estímulo constante desses profissionais, já que eles pos- 
suem contato diário e próximo com os alunos, e podem auxiliar no processo de promoção de saúde, de modo a influenciar as crianças na construção de hábitos saudáveis (PAES; PAIXÃO, 2016). Professores com conhecimento sobre educação em saúde bucal são mais preparados para instruírem tanto as crianças quanto os pais/responsáveis sobre questões nutricionais e hábitos de higiene bucal (KHURANA et al., 2020; CARDOSO et al., 2019).

Com o advento da pandemia da COVID-19 e a paralisação das atividades escolares presenciais, o projeto de extensão nos moldes realizados durante os anos de 2018 a 2020 tornou-se impraticável, mas ações educativas remotas foram propostas para se manter o vínculo entre os participantes do projeto e alunos, pais e professores da CMEI. Isso foi possível devido à capacidade que a extensão possui de se moldar às necessidades do contexto em que se insere, mostrando o potencial de docentes e discentes extensionistas em readaptar-se na execução das ações desenvolvidas no compromisso com a sociedade (NASCIMENTO et al., 2020; POST et al., 2021).

Desta forma, frente à impossibilidade de realização de atividades educativas presenciais, a utilização de ferramentas digitais tornou-se o principal meio para disseminação e compartilhamento de conteúdo educativo na abordagem individual e coletiva da população (SERR ̃̃O, 2020; TOMAZ, 2020; FARIA et al., 2020; OLIVEIRA; DEGGAU, 2021). Durante o período de afastamento físico e isolamento social, observou-se como fundamental a construção de tecnologia educativa para que haja a promoção em saúde, visto que a manutenção da saúde bucal influencia diretamente na saúde geral do indivíduo (SILVA et al., 2021; MONTANDON, 2021).

Várias estratégias no campo da saúde bucal, neste momento de pandemia, estão sendo discutidas e implementadas no Brasil e no mundo, de forma manter o cuidado ativo com a saúde bucal (CASTRO, 2021). No entanto, há obstáculos, assim como destacado por Oliveira et al. (2020), que relatam que uma desvantagem da abordagem a distância é a não garantia de que o conteúdo chegue a todas as famílias, visto que alguns grupos não possuem acesso à internet.

A mensuração do impacto do compartilhamento de informações por meio de vídeos e cartilhas é de difícil realização, pois esbarra em características próprias da família, que vão desde a organização familiar até o acesso à internet. Há carência de pesquisas científicas delineadas e conduzidas especificamente para este fim, além do prejuízo no estabelecimento de vínculos diretos entre o profissional de saúde e as crianças, aspecto importante, pois, como relatado por Rodrigues et al. (2020), o contato frequente desmistifica a imagem do cirurgiãodentista como um profissional que causa dor, fazendo com que as crianças se sintam mais confortáveis em ir regularmente à unidade de saúde e fazer o tratamento odontológico. 


\section{Conclusão}

Práticas de educação em saúde em CMEIS são necessárias, visto que esses lugares permitem sensibilizar crianças, professores e responsáveis quanto à promoção de saúde e a prevenção de doenças e agravos bucais, de maneira a melhorar a qualidade de vida dos envolvidos, perpetuando comportamento e hábitos saudáveis.

As restrições impostas pela pandemia da COVID-19 tornaram necessárias mudanças metodológicas na abordagem do projeto de extensão, permitindo a diversificação das ações promovidas com opções a serem introduzidas como práticas rotineiras futuramente. A participação em projeto de extensão oportunizou aos acadêmicos uma formação profissional e pessoal, com experiências enriquecedoras para atuar frente às demandas que a sociedade apresenta.

A participação em um projeto de extensão possibilitou que alunos do curso de graduação em Odontologia atuassem de maneira direta com ações de educação e prevenção em saúde bucal, e estimulou a autonomia dos alunos quando houve a necessidade de modificação das ações práticas, em decorrência do isolamento social e interrupção das atividades escolares. O estímulo a esse tipo de prática incentiva a formação profissional e a responsabilização perante a sociedade.

\section{Agradecimentos}

Os autores agradecem à Universidade Estadual de Londrina (UEL), à Pró-Reitoria de Extensão, Cultura e Sociedade (PROEX-UEL), a qual o projeto está vinculado, à Fundação Araucária e Superintendência de Ciência, Tecnologia e Ensino Superior (SETI), pelo financiamento de bolsas de ação extensionistas.

\section{Referências:}

BRASIL. Ministério da Educação. Portaria No 343, de 17 de março de 2020. Dispõe sobre a substituição das aulas presenciais por aulas em meios digitais enquanto durar a situação de pandemia do Novo Coronavírus - COVID-19. 2020. Disponível em: http://www.in.gov.br/en/web/ dou/- /portaria-n-343-de-17-de-marco-de-2020- 248564376. Acesso em: 12 ago. 2021.

CAMPOS, B.H. et al. Telessaúde e telemedicina: uma ação de extensão durante a pandemia. Revista Aproximação, v. 2, n. 4, 2020. 
CARDOSO, A.T.G. et al. Experiência de educação em saúde bucal em escola de educação infantil na República de Cabo Verde, África. Arch. Health Invest, v.8, n.5, p. 267-270, 2019.

CASTRO, R. et al. Possibilidades em um projeto de extensão de apoio ao programa saúde na escola frente ao contexto da Covid-19. Expressa Extensão, v. 26, n. 1, p. 84-93, 2021.

MONTEIRO, R. C.; CASTRO, A.L.S. Educação continuada em saúde bucal para professores da educação infantil: contexto atual e importância para a odontologia preventiva. Revista Eletrônica Acervo Odontológico, v. 3, p. 6082-6082, 2021.

FARIA, M.H.D. et al. Relato de alunos de odontologia no enfrentamento à Covid-19. Cadernos ESP-Revista Científica da Escola de Saúde Pública do Ceará, v. 14, n. 1, p. 118-122, 2020.

FIRMINO, R.T. et al. Is parental oral health literacy a predictor of children's oral health outcomes? Systematic review of the literature. International journal of paediatric dentistry, v. 28, n. 5, p. 459-471, 2018.

OLIVEIRA, M. F.; DEGGAU, R.B. A extensão universitária na Odontologia durante a pandemia do COVID-19-relato de experiência. Extensão em Foco, n. 23, 2021.

KHURANA, C. et al. Effectiveness of an oral health training program for schoolteachers in India: An interventional study. Journal of Education and Health Promotion, v. 9, 2020.

KUPFERSCHMIDT, K.; COHEN, J. Can China's COVID-19 strategy work elsewhere? Science, v. 367, n. 6482, p. 1061-1062, 2020.

LAI, H.F. et al. Parental oral health literacy of children with severe early childhood caries in Hong Kong. Eur J Paediatr Dent, Italy, v. 18, n. 4, p.326-31, 2017.

DE MELO, M.M.D.C. et al. Relato de experiências discentes do núcleo de saúde bucal integrante do projeto de extensão "Enfrentamento ao Covid19: produzir saúde e defender da vida”. Revista Capim Dourado - Diálogos em Extensão, v. 3, n. 2, p. 170-181, 2020.

MELO, M. C.; FONSECA, C. M. F.; VASCONCELLOS-SILVA, P. R. Internet e mídias sociais na educação em saúde: o cenário oncológico. Cadernos do Tempo Presente, v. 27, p. 69-83, 2017. 
MERCURI, E. et al. Contactless: a new personalised telehealth model in chronic pediatric diseases and disability during the COVID-19 era. Italian Journal of Pediatrics, v. 47, n.1, p.29, 2021.

MONTES, G.R. et al. Caregiver's oral health literacy is associated with prevalence of untreated dental caries in preschool children. Cien Saude Colet, v. 24, n.7, p. 2737-2744, 2019.

MONTANDON, F.M. et al. O Instagram ${ }^{\circledR}$ como ferramenta de educação e multiplicação do conhecimento em saúde bucal. Revista Com Censo: Estudos Educacionais do Distrito Federal, v. 7, n. 4, p. 185-189, 2020.

NASCIMENTO, F.G.M. et al. Uso do Jogo Plague Inc.: uma possibilidade para o Ensino de Ciências em tempos da COVID-19. Braz. J. of Develop., v. 6, n. 5, p. 25909-25928, 2020.

NUNES GP, et al. Evaluation of Dental Caries Behavior in Childhood: Health Promotion and Control. Journal of Health Sciences, v. 21 n. 5: p. 500-503, 2019.

NUNES, V.H.; PEROSA, G.B. Cárie dentária em crianças de 5 anos: fatores sociodemográficos, lócus de controle e atitudes parentais. Ciência \& Saúde Coletiva, v. 22, p. 191-200, 2017.

OLIVEIRA, G.T. et al. Promoção de saúde por meios digitais durante a pandemia da Covid-19 em um projeto de extensão em Disfagia. Raízes e Rumos, v. 8, n. 2, p. 296-306, 2020.

RODRIGUES, A.A.A.O. et al. Capacitação dos professores do ensino infantil para promoção da saúde bucal de pré-escolares. Expressa Extensão, v. 25, n. 3, p. 358-366, 2020.

PAES, C.C.D.C.; PAIXÃO, A.N.P. A importância da abordagem da educação em saúde: revisão de literatura. Revista de Educação da Universidade Federal do Vale do São Francisco, v. 6, n. 11, 2016.

POST, L.K. et al. Projeto de extensão cetat em tempo de pandemia: novas experiências e reflexões. Expressa Extensão, v. 26, n. 1, p. 163-171, 2021. 
SERRÃO, A.C.P. Em tempos de exceção como fazer extensão? Reflexões sobre a Prática da Extensão Universitária no Combate à Covid-19. Revista Práticas em Extensão, v. 4, n. 1, p. 47-49, 2020.

SIGAUD, C.H.S. et al. Promoción de la salud bucal de niños preescolares: efectos de una intervención educativa lúdica. Revista Brasileira de Enfermagem, v. 70, n. 3, p. 519-525, 2017.

SILVA, Ana Flávia Hott et al. Projeto sorrindo na pandemia. Anais da Noite Acadêmica, v. 1, n. 1, 2021.

TOMAZ, J.B.C. Educação na saúde em tempos de pandemia: desafios e oportunidades. Cadernos ESP-Revista Científica da Escola de Saúde Pública do Ceará, v. 14, n. 2, p. 7-9, 2020.

WIERSINGA, W.J. et al. Pathophysiology, transmission, diagnosis, and treatment of coronavirus disease 2019 (COVID-19): a review. Jama, v. 324, n. 8, p. 782-793, 2020. 